\title{
Emergencia sanitaria: dos marcos de deliberación*
}

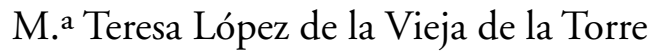 \\ Universidad de Salamanca \\ tlv@usal.es
}

David Rodríguez-Arias Vailhen

Universidad de Granada

dra@ugr.es

Fecha de recepción: 3-6-2020

Fecha de aceptación: 30-6-2020

\section{Resumen}

La Organización Mundial de la Salud ha admitido que el acceso a la atención sanitaria se ha visto seriamente afectado por la COVID-19. Su impacto ha forzado a distribuir los recursos y a establecer prioridades que sean justificables. Un informe del Ministerio de Sanidad español ha reiterado la importancia de la ética en tal situación, y otras entidades han hecho recomendaciones para los profesionales de la salud. No resulta sencillo tomar decisiones en situaciones de escasez sobrevenida; tampoco llegar al equilibrio entre los principios de equidad y eficiencia. El objetivo del artículo es considerar estos niveles: (1) la toma de decisiones en situación de emergencia sanitaria, (2) el marco de los principios, con la equidad para la distribución y la eficiencia para la gestión de los recursos, y (3) la deliberación como proceso apropiado para la solución de conflictos, porque favorece la transparencia y la rendición de cuentas en las políticas públicas, incluida la política sanitaria.

Palabras clave: COVID-19; salud pública; principios; deliberación

\section{Abstract. Health emergency: Two frameworks for deliberation}

Access to healthcare has been heavily affected by the COVID-19, according to the World Health Organization. Its impact has forced the distribution of resources and the need for justifiable priority-setting. A report published by the Spanish Ministry of Health has reiterated the importance of ethics in such a situation, and other entities have made recommendations for health professionals. However, it is not easy to make decisions in situations of unexpected scarcity, nor is it possible to reach a balance between equity and efficiency. Against this backdrop, the objective of the article is to consider the following levels: (1) decision making in a health emergency situation; (2) the framework of principles, with the equitable distribution and efficient management of resources, and (3) deliberation as a appropriate procedure for conflict resolution and as a process that fosters public policy transparency and accountability in public policies, including health policy.

Keywords: COVID-19; public health; principles; deliberation

* Trabajo realizado en el marco de los proyectos de I+D BIOCIT (PID2019-105935RB-I00) $<$ https://bioeticayciudadania.weebly.com/presentacioacuten.html> e INEDyTO (FFI201788913-P) <https://www.inedyto.com/>. 


\section{Sumario}
1. Introducción
5. Conclusiones
2. La toma de decisiones
Referencias bibliográficas
3. Un marco de principios
4. Rendición de cuentas y deliberación pública

\section{Introducción}

En mayo de 2020 la Organización Mundial de la Salud llegó a admitir que el acceso a la atención sanitaria se había visto gravemente afectado durante la crisis generada por la COVID-19. El impacto de la pandemia en las organizaciones de salud ha obligado a distribuir aquellos recursos que estuvieran disponibles y a establecer algunas prioridades de manera justificable. En tal situación las recomendaciones para los profesionales de la salud deben realizarse sobre la base de criterios éticos y no solo técnicos. Un informe del Ministerio de Sanidad español reiteraba la importancia de la ética en tal circunstancia: la equidad, la no discriminación y la solidaridad han de guiar todas las actuaciones. Otras entidades han hecho recomendaciones para los profesionales de la salud. En casi todos los casos se planteaba la necesidad y, a la vez, la dificultad de llegar a un equilibrio entre el principio de equidad y el uso eficiente de recursos en beneficio de un mayor número de pacientes.

La pandemia producida por el virus SARS-CoV2 ha obligado a los centros sanitarios de todo el país a aplicar criterios de priorización. De manera particularmente problemática, la afluencia masiva a los servicios de cuidados intensivos (UCI) de pacientes afectados por la COVID-19 y con insuficiencia respiratoria ha generado la necesidad de racionar camas de cuidados intensivos y dispositivos de ventilación mecánica asistida. La previsión es que sean necesarias nuevas medidas de priorización cada vez que esté en juego la asignación de vacunas y tratamientos cuya oferta quede superada por la demanda. Sin embargo, no resulta sencillo establecer el equilibrio entre valores y marcos normativos diferentes.

Por eso es necesario considerar todos los posibles argumentos. Es decir, hace falta un ejercicio de deliberación. La equidad es el principio básico para la salud pública, su significado y alcance han sido explicados por distintas teorías de la justicia. La eficiencia figura entre los principios de gobernanza para las políticas. El reto consiste en armonizar ambos principios y mostrar que los enfoques de salud pública y de economía de la salud pueden ser complementarios. El objetivo del artículo es considerar los niveles siguientes: la toma de decisiones en la pandemia; el marco cognitivo y normativo que estructura tales decisiones mediante dos principios, equidad para la distribución y eficiencia para la gestión de los recursos; y la deliberación, entendida como un procedimiento que consiste en ponderar argumentos con igual consideración 
y respeto, para debatir con otros y, al final, llegar a acuerdos que sean consistentes. Defenderemos que la deliberación resulta adecuada para la solución de conflictos, a la vez que favorece la transparencia y la rendición de cuentas de la política sanitaria.

\section{La toma de decisiones}

La escasez de recursos sanitarios, motivada por la crisis de salud pública, ha sacado a la luz problemas éticos que cobran una urgencia y una relevancia especiales. ¿A quién debe priorizarse y por qué? ¿Cómo conseguir que la distribución de los recursos sea justa? ¿Qué principios y criterios deben guiar el racionamiento de medios limitados? ¿Cómo optimizar esos recursos y cómo valorar su mejor aprovechamiento? ¿Deberían priorizarse ciertos grupos de pacientes por su estado de salud o por su «valor social o profesional»? Según se pregunta Rueda (2020), ¿deberían ser automáticamente excluidos ciertos grupos? ¿Cómo evitar formas inaceptables de discriminación? ¿Qué conocimientos y procedimientos son necesarios para responder a estas cuestiones?

Cualesquiera que fueran las medidas que se adoptasen, implicarían necesariamente una toma de decisiones sobre valores, por lo que pueden propiciar desacuerdos y generar controversia pública. Las respuestas motivadas que se ofrecieran contribuirían, por un lado, a conseguir que las medidas de salud pública adoptadas, pudiendo ser cuestionables, fueran más legítimas. Por otro lado, situarían a los profesionales sanitarios y al conjunto de la sociedad en mejores condiciones para afrontar los efectos de la crisis. La deliberación resulta, por lo tanto, un paso ineludible para la gobernanza de crisis sanitarias como la generada por la pandemia de COVID-19. Las razones serían las siguientes:

En todo ejercicio de priorización - también en las prácticas de racionamiento- deben ponderarse valores en conflicto, como la eficiencia, la equidad, la protección frente al daño, la dignidad, el valor instrumental, la solidaridad, la confianza, la no discriminación y la reciprocidad (Smith y Upshur, 2019). Cada uno de ellos, considerados por separado, tiene gran importancia política y social. Contribuyen al cumplimiento de objetivos ampliamente compartidos, como la convivencia, la justicia o la seguridad. Sin embargo, en la práctica no es posible maximizarlos todos simultáneamente. Esto se observa con claridad cuando se trata de racionar recursos limitados. Los criterios de asignación, o bien excluyen a ciertas categorías de pacientes del reparto, o bien establecen un orden entre varios pacientes (por ejemplo, asignando un baremo de supervivencia en cuidados intensivos), o bien sirven para desempatar (por ejemplo, entre dos personas con igual probabilidad de supervivencia). En cualquiera de estos casos el criterio implica una jerarquización de los valores en juego: prioriza el cumplimiento de algunos y pospone o incluso sacrifica el de otros.

Determinar qué valores son más importantes no es una cuestión sencilla. Como en otros debates sobre salud, se producen en este caso desacuerdos sustantivos, muchas veces irreconciliables, entre puntos de vista razonables. 
Durante ese proceso de justificación surgen inevitablemente ciertas preguntas: ¿cómo priorizar los principios y los valores morales que preceden a la identificación de los criterios? ¿Quién tiene autoridad sobre estas cuestiones esencialmente normativas? ¿Qué papel les corresponde a los expertos y cuál a los no expertos en la concreción de esas políticas?

\section{Un marco de principios}

Los equipos sanitarios adoptan decisiones clínicas sobre priorización y racionamiento de recursos sanitarios. Al hacerlo aplican criterios y se basan en consideraciones técnicas (por ejemplo, sobre la morbilidad y la probabilidad de recuperación y la esperanza de vida de los pacientes) acerca de las que tienen pericia profesional y autoridad epistémica. Sin embargo, la justificación y la ordenación de los criterios no es una cuestión meramente técnica. Esto se debe a que la identificación y la ponderación de los valores que subyacen a la definición de esos criterios no requieren juicios meramente técnicos, sino de valor. Las ciencias de la salud pueden estimar con precisión cuál es la posibilidad de mejora de varios pacientes, pero ningún juicio técnico muestra que el orden de llegada deba ser decisivo, cómo elegir entre urgencia y beneficio esperado o si se debe priorizar a quien podrá vivir más años sobre aquellas personas que vivirán durante menos tiempo, pero con más calidad de vida. Las competencias profesionales de los sanitarios, como las vinculadas al diagnóstico y al pronóstico de enfermos, resultan esenciales y deben contemplarse en la asignación de recursos, pero no sirven para justificar la elección ni la ordenación de los criterios de priorización. La pericia técnica de profesionales de la salud no es autoritativa para determinar el modo de jerarquizar valores como la esperanza de vida, el número de personas salvadas, la utilidad instrumental o la urgencia.

Los marcos cognitivos y normativos que sirven de fundamento no son competencia exclusiva de los expertos (López de la Vieja, 2002). Diferentes propuestas de distribución de recursos y formas de establecer prioridad entre unos pacientes y otros resultan de conceder mayor o menor importancia a la equidad o a la eficiencia. Se trata de categorías diferentes y, además, de "marcos» — cognitivos y normativos (Campbell, 2002) — , en el sentido de esquemas o filtros. Estructuran la información, los valores (Scheuffele y Tewksbury, 2007) y definen qué asuntos son relevantes.

a) La equidad es el principio básico en materia de salud pública (Ley 33/2011, art. 3 a). Se refiere al modo en que se distribuyen los bienes y las oportunidades, y exige que demandas comparables reciban una atención comparable. Las teorías de la justicia han explicado su significado (Rawls, 2001: 50-58) y su alcance para cuestiones de salud (Daniels, 1982; Daniels y Sabin, 2002). De manera genérica, la equidad indica que la asignación de recursos sanitarios tenga en cuenta diferencias relevantes entre los destinatarios, sin incurrir en formas de discriminación que agraven las desigualdades ya existentes. La Organización Mundial de la Salud aplica este prin- 
cipio al contexto de las crisis, como las provocadas por una pandemia, e invoca la equidad como el principio que exigiría lo siguiente: $1^{\circ}$. Tratar de forma análoga casos análogos; $2^{\circ}$. Evitar cualquier forma de discriminación o explotación, y $3^{\circ}$. Ser sensible con respecto a las personas especialmente vulnerables al daño o la injusticia (WHO, 2016: 8).

b) La eficiencia no se define por el modo en que se distribuyen los recursos, sino por los resultados que se obtienen con ellos. Consiste en el mejor aprovechamiento de los medios, es decir, en llegar a los mejores resultados con la menor inversión de recursos. Figura entre los principios de gobernanza o buen gobierno (Commission of the European Communities, 2001: 8). Esta definición formal de la eficiencia —al igual que sucede con la que ya se ha ofrecido de la equidad - puede concretarse en diferentes aplicaciones en función de cuál sea su contenido. En particular, dependerá del objetivo o de los resultados más valorados. Pese a sus diferencias, sería deseable que hubiera cierto equilibrio (Culyer, 2006) o complementariedad entre ambos principios o, lo que es lo mismo, entre dos enfoques diferentes: salud pública y economía de la salud. ¿Cuál sería el método?

Si los principios marcan las líneas generales de actuación con carácter regulativo (Beauchamp, 2010: 157), la deliberación es una forma de comunicación enfocada a la solución de conflictos (Mansbridge, 2015: 27-50). El procedimiento consiste en sopesar los distintos argumentos con igual consideración y respeto para debatir con otros y, al final, llegar a acuerdos que sean consistentes (Fishkin, 2009: 1-31, 95-105). En el ámbito público sirve para dar y pedir razón de las decisiones, siempre que la información correspondiente y el procedimiento mismo hayan sido accesibles para la ciudadanía (Gutmann y Thompson, 2004: 1-62), es decir, se trata de un proceso para crear acuerdos y poder justificar las políticas de cada gobierno (Cohen, 2009).

\section{Rendición de cuentas y deliberación pública}

Durante la crisis provocada por la COVID-19 la ciudadanía ha sido testigo de la presentación de un cierto número de protocolos y recomendaciones sobre priorización, racionamiento de recursos y triaje de pacientes. No debería extrañar que las sociedades contemporáneas, plurales, ofrezcan distintas soluciones razonables - aunque no necesariamente compatibles entre sí- ante problemas similares. En algunos casos, el contexto explica - y justifica- esas diferencias. Por ejemplo, los criterios de priorización no deberían ser los mismos en hospitales que sufran la afluencia de pacientes en grados muy distintos, porque la planificación de los tratamientos es más realizable cuando las hospitalizaciones se producen de manera progresiva que cuando se producen de manera abrupta. En efecto, factores como la urgencia, la concentración de la demanda, la tasa de letalidad o la disponibilidad de equipos sanitarios en cada región pueden justificar políticas diferentes. 
La equidad exige atender de manera análoga — no necesariamente idéntica- demandas análogas. La pretensión de universalizabilidad, implícita en la noción de equidad, exige también que las diferencias locales estén debidamente justificadas. Durante la crisis que ha vivido el país, este no siempre ha sido el caso. Más allá de diferencias contextuales, se han producido desacuerdos profundos sobre los valores fundamentales en la asignación, sobre la oportunidad de emplear criterios categóricos de exclusión y umbrales de edad, o sobre la importancia de atender a la discapacidad (Ministerio de Sanidad, 2020; Comité de Bioética de España, 2020; SEMICYUC, 2020).

Las controversias normativas en materia de salud son comunes, y han sido analizadas en bioética desde hace décadas. La bioética ha desarrollado procedimientos para justificar decisiones difíciles. En el contexto específico del racionamiento destacan dos: la rendición de cuentas (Daniels, 2000) y la deliberación pública (Neblo, 2007).

La rendición de cuentas sobre la razonabilidad de las decisiones (accountability for reasonableness) es el método propuesto por Daniels y Sabin (2008) para justificar el establecimiento de límites al tratamiento y a las prestaciones de los servicios de salud. Se trata de un procedimiento que exige someter esas decisiones al escrutinio público, con procesos transparentes, suficientemente razonados y abiertos a la revisión, además de:

1. Asegurar accesibilidad pública a las decisiones que se adopten, en condiciones de transparencia y publicidad.

2. Ofrecer para esas decisiones al menos una justificación adecuada basada en razones que todas las personas — también las minorías - puedan considerar justificables en un contexto de cooperación.

3. Habilitar mecanismos públicos de revisión y crítica de las decisiones adoptadas.

4. Evaluar el cumplimiento de los tres puntos anteriores.

Un ejemplo de este procedimiento lo constituyen los protocolos de priorización de recursos de las UCI, que incluyen la incorporación de mecanismos de revisión y reclamación (White et al., 2020).

La deliberación pública es un proceso a través del cual se involucra al público en una discusión estructurada y constructiva, con capacidad de informar cualificadamente la toma de decisiones sobre políticas públicas (Neblo, 2007; Blacksher et al., 2012). Existe un precedente de deliberación pública con respecto a los criterios de racionamiento en circunstancias de epidemia (Daugherty Biddison et al., 2014; Daugherty Biddison et al., 2018; Daugherty Biddison et al., 2019) que sugiere que los valores del público constituyen una información necesaria y útil para elegir entre múltiples enfoques éticamente permisibles. 


\section{Conclusiones}

La incorporación del punto de vista de los no expertos en las decisiones sobre la asignación de recursos vitales limitados es necesaria, porque el público se verá afectado por las consecuencias de esas decisiones y porque todo intento exitoso de asignación requiere confianza y cooperación por parte de la ciudadanía. Esta solo podrá asumir como propias las políticas sanitarias si se facilitan formas inclusivas de participación y de deliberación públicas.

Además, el conocimiento de cuál es la perspectiva ciudadana sobre estos asuntos prepara mejor a las autoridades de salud para desarrollar guías adecuadas de asignación de recursos y para comunicar y defender políticas que puedan ser controvertidas. En conclusión, el procedimiento de deliberación no solo aporta legitimidad y favorece la rendición de cuentas en las políticas públicas, equitativas y eficientes en la gestión de catástrofes, sino que hacerlo así apuntala la confianza del público en las instituciones.

\section{Referencias bibliográficas}

Beauchamp, Tom (2010). Standing on Principles. Oxford: Oxford University Press. Blacksher, Erika; Diebel, Alice; Forest, Pierre-Gerlier; Dorr Goold, Susan y Abelson, Julia (2012). "What Is Public Deliberation?». Hastings Center Report, 42 (2), 14-16. $<$ https://doi.org/10.1002/hast.26>

Campbell, John (2002). «Ideas, Politics, and Public Policy». Annual Review of Sociology, 28, 21-38. <https://doi.org/10.1146/annurev.soc.28.110601.141111>

Cohen, Joshua (2009). «Truth and Public Reason». Philosophy \& Pubklic Affairs, 37, 2-42. $<$ https://doi.org/10.1111/j.1088-4963.2008.01144.x>

Comité DE BioÉTiCA DE España (2020). Informe del Comité sobre los aspectos bioéticos de la priorización de recursos sanitarios en el contexto de la crisis del Coronavirus. Recuperado el 2 de junio de 2020, de <http://assets.comite debioetica.es/files/documentacion/Informe\%20CBE-\%20Priorizacion\%20 de\%20recursos\%20sanitarios-coronavirus\%20CBE.pdf>.

Commission of the European Communities (2001). «European Governance-A White Paper». COM, 428 ( 25 de julio). Recuperado el 2 de junio de 2020, de <https://ec.europa.eu/commission/presscorner/detail/en/ DOC_01_10>.

Culyer, Anthony (2006). «The Bogus Conflict between Efficiency and Vertical Equity». Health Economics, 15, 1155-1158. $<$ https://doi.org/10.1002/hec.1158>

Daniels, Norman (1982). «Equity of Access to Health Care: Some Conceptual and Ethical Issues». The Milkbank Quarterly, 60, 51-81. Recuperado el 2 de junio de 2020, de <https://www.milbank.org/quarterly/articles/ equity-of-access-to-health-care-some-conceptual-and-ethical-issues/>. 
- (2000). «Accountability for Reasonableness». BMJ: British Medical Journal, 321 (7272), 1300-1301. <https://doi.org/10.1136/bmj.321.7272.1300>

Daniels, Norman y Sabin, James E. (2008). Setting Limits Fairly: Can We Learn to Share Medical Resources? Setting Limits Fairly. Oxford: Oxford University Press. <https://doi.org/10.1093/acprof:oso/9780195149364.001.0001>

Daugherty Biddison, Elizabeth L.; Gwon, Howard; SсHOCh-Spana, Monica; Cavalier, Robert; White, Douglas B.; Dawson, Timothy; Terry, Peter B. et al. (2014). «The Community Speaks: Understanding Ethical Values in Allocation of Scarce Lifesaving Resources during Disasters». Annals of the American Thoracic Society, 11 (5), 777-783. $<$ https://doi.org/10.1513/AnnalsATS.201310-379OC>

Daugherty Biddison, E. Lee; Faden, Ruth; Gwon, Howard S.; Mareiniss, Darren P.; Regenderg, Alan C.; Sсhoch-Spana, Monica; Schwartz, Jack y Toner, Eric S. (2019). «Too Many Patients...: A Framework to Guide Statewide Allocation of Scarce Mechanical Ventilation During Disasters». Chest, 155 (4), 848-854. <https://doi.org/10.1016/j.chest.2018.09.025>

Daugherty Biddison, E. Lee; Gwon, Howard S.; Schoch-Spana, Monica; Regenberg, Alan C.; Juliano, Chrissie; Faden, Ruth R. y Toner, Eric S. (2018). «Scarce Resource Allocation During Disasters: A Mixed-Method Community Engagement Study». Chest, 153 (1), 187-195. <https://doi.org/10.1016/j.chest.2017.08.001>

Fishrin, James (2009). When People Speaks. Oxford: Oxford University Press. Gutmann, Amy y Thompson, Denis (2004). Why Deliberative Democracy? Princeton: Princeton University Press.

«Ley 33/2011, de 4 de octubre, General de Salud Pública». Boletín Oficial del Estado, 240 (5 de octubre). Recuperado el 2 de junio de 2020, de <https:// www.boe.es/eli/es/1/2011/10/04/33/con>.

López de LA VIEJA, M. ${ }^{a}$ Teresa (2002). “"Expertos” en bioética». Isegoría, 24, 167-180. <https://doi.org/10.3989/isegoria.2002.i27.560>

Mansbridge, Jane (2015). «A Minimalist Definition of Deliberation». En: Heller, Patrick y Rao, Vijayendra (eds.). Deliberation and Development. Washington: World Bank Group.

Ministerio de Sanidad (2020). Informe del Ministerio de Sanidad sobre los aspectos éticos en situaciones de pandemia: El SARS-CoV-2. Recuperado el 2 de junio de 2020, de <https://www.mscbs.gob.es/profesionales/saludPu blica/ccayes/alertasActual/nCov-China/documentos/AspectosEticos_en_ situaciones_de_pandemia.pdf $>$.

Neblo, Michael A. (2007). «Family Disputes: Diversity in Defining and Measuring Deliberation». Swiss Political Science Review, 13 (4), 527-557. <https://doi.org/10.1002/j.1662-6370.2007.tb00088.x>

Rawls, John (2001). Justice as Fairness: A Restatement. Cambridge: Harvard University Press. 
Rueda, Jon (2020). «¿No es país para viejos?: La edad como criterio ético de triaje durante la pandemia de la COVID-19». Enrahonar, 65, 85-98.

Scheuffele, Dietram y Tewksbury, David (200). «Framing Agenda Setting, and Priming: The Evolution of Three Media Effects Models». Journal of Communication, 57, 9-20. <https://doi.org/10.1111/j.0021-9916.2007.00326.x>

SEMICYUC (Sociedad Española de Medicina InTensiva, Crítica y UniDAdes Coronarias) (2020). Recomendaciones éticas para la toma de decisiones en la situación excepcional de crisis por la pandemia COVID-19 en las unidades de cuidados intensivos. Recuperado el 2 de junio de 2020, de $<$ https://semicyuc.org/wp-content/uploads/2020/03/\%C3\%89tica_SEMI CYUC-COVID-19.pdf>.

Smith, Maxwell y Upshur, Ross (2019). "Pandemic Disease, Public Health, and Ethics». En: The Oxford Handbook of Public Health Ethics (9 de septiembre). <https://doi.org/10.1093/oxfordhb/9780190245191.013.69>

White, Douglas; Katz, Mitchell; Luce, John; Lo, Bernard; Daugherty BidDison, Lee; Toner, Eric y Halpern, Scott (2020). Allocation of Scarce Critical Care Resources During a Public Health Emergency: Executive Summary. University of Pittsburgh. Department of Critical Care Medicine. Recuperado el 2 de junio de 2020, de <https://ccm.pitt.edu/sites/default/ files/UnivPittsburgh_ModelHospitalResourcePolicy_2020_04_15.pdf>.

WHO (2016). Guidance for Managing Ethical Issues in Infectious Disease Outbreaks. Recuperado el 2 de junio de 2020, <https://www.who.int/ethics/ publications/infectious-disease-outbreaks/en/>.

- (2019). Ethics and COVID-19: Resource Allocation and Priority-Setting. Recuperado el 2 de junio de 2020, <https:/www.who.int/ethics/publica tions/ethics-and-covid-19-resource-allocation-and-priority-setting/en/> 
M. ${ }^{a}$ Teresa López de la Vieja de la Torre es catedrática emérita de Filosofía Moral en la Universidad de Salamanca. Profesora adjunta del Departamento de Bioética de la Case Western Reserve University, de Cleveland. Vocal del Comité de Bioética de la Universidad de Salamanca, vocal del Comité de Ética de la Diputación de Salamanca. Investigadora principal de Proyectos de I+D. Algunas de sus publicaciones son: Principios morales y casos prácticos, Madrid, Tecnos, 2000; Ética y literatura, Madrid, Tecnos, 2003; La mitad del mundo: Ética y critica feminista, Salamanca, Universidad de Salamanca, 2004; Bioética y ciudadanía, Madrid, Biblioteca Nueva, 2008; La pendiente resbaladiza: La práctica de la argumentación moral, Madrid, Plaza y Valdés, 2011; Bioética y literatura, Madrid, Plaza y Valdés, 2013; «Bioethics \& Internet», en Ten Have, H. (ed.), Encyclopedia of Global Bioethics, Dordrecht, Springer, 2015, p. 1-12, DOI: 10.1007/978-3-319-05544-2_257-1; además ha editado Bioética: Presente futuro, Madrid, Plaza y Valdés, 2019.

M. ${ }^{a}$ Teresa López de la Vieja de la Torre is Emeritus Professor of moral philosophy at the University of Salamanca and Adjunct Professor in the Department of Bioethics of Case Western Reserve University, Cleveland. She is also a member of the Bioethics Committee of the University of Salamanca and a member of the Ethics Committee of the Regional Council of Salamanca. She is the principal investigator of $R \& D$ projects. Her publications include Principios morales y casos prácticos, Tecnos, Madrid, 2000; Ética y literatura, Tecnos, Madrid, 2003; La mitad del mundo: Ética y critica feminista, University of Salamanca, Salamanca, 2004; Bioética y ciudadanía, New Library, Madrid, 2008; La pendiente resbaladiza: La práctica de la argumentación moral, Plaza and Valdés, Madrid, 2011; Bioética y literatura, Plaza and Valdés, Madrid, 2013; and "Bioethics \& Internet" in Ten Have, H. (ed.), Encyclopedia of Global Bioethics, Springer, Dordrecht, 2015, pp. 1-12. She is also the editor of Bioética: Presente futuro, Plaza y Valdés, Madrid, 2019.

David Rodríguez-Arias Vailhen es investigador Ramón y Cajal en la Universidad de Granada, subdirector de la Unidad Científica de Excelencia FiloLab-UGR (Área de Ética, Departamento de Filosofía I) y vocal del Comité de Bioética de Andalucía. Se doctoró en Filosofía Moral y en Ética Médica (Universidad de Salamanca y Université Paris-Descartes). Sus investigaciones se han centrado en la bioética clínica (final de la vida, trasplantes) y global, la neuroética y la ética de la investigación, y tienen un enfoque analítico y empírico. Publicaciones recientes: "Examining Public Trust in Categorical versus Comprehensive Triage Criteria», The American Journal of Bioethics, 20 (7), 106-109 (27 de julio de 2020); «Death determination and clinicians' epistemic authority», The American Journal of Bioethics, 20 (6), 44-47 (22 de mayo de 2020); «How Do People Use 'Killing', 'Letting Die' and Related Bioethical Concepts?: Contrasting Descriptive and Normative Hypotheses», Bioethics (13 de enero de 2020), y "The Dead Donor Rule as Policy Indoctrination», The Hastings Center Report (25 de diciembre de 2018).

David Rodríguez-Arias Vailhen is a Ramón y Cajal researcher at the University of Granada, deputy director of the FiloLab-UGR Scientific Unit of Excellence (Ethics Area, Department of Philosophy I) and member of the Andalusian Bioethics Committee. He completed a PhD in Moral Philosophy and Medical Ethics from the University of Salamanca and Université Paris-Descartes. His research has focused on clinical (end of life, transplantation) and global bioethics, neuroethics and research ethics, and has an analytical and empirical approach. His recent publications include "Examining public trust in categorical versus comprehensive triage criteria", The American Journal of Bioethics, 20 (7), 106-109 (July 27, 2020); "Death determination and clinicians' epistemic authority," The American Journal of Bioethics, 20 (6), 44-47 (May 22, 2020); "How do people use 'killing', 'letting die' and related bioethical concepts?: Contrasting descriptive and normative hypotheses", Bioethics (January 13, 2020), and "The Dead Donor Rule as Policy Indoctrination", The Hastings Center Report (December 25, 2018). 